\title{
Mutant PKC $\gamma$ in Spinocerebellar Ataxia Type 14 Disrupts Synapse Elimination and Long-Term Depression in Purkinje Cells In Vivo
}

\author{
Anton N. Shuvaev, ${ }^{1,2}$ Hajime Horiuchi, ${ }^{1}$ Takahiro Seki, ${ }^{3}$ Hanna Goenawan, ${ }^{1}$ Tomohiko Irie, ${ }^{1}$ Akira Iizuka, ${ }^{1}$ Norio Sakai, ${ }^{3}$ \\ and Hirokazu Hirai ${ }^{1}$ \\ ${ }^{1}$ Department of Neurophysiology, Gunma University Graduate School of Medicine, Maebashi, Gunma 371-8511, Japan, ${ }^{2}$ Department of Neurosurgery and \\ Neurology, Krasnoyarsk State Medical University, Krasnoyarsk 660022, Russia, and ${ }^{3}$ Department of Molecular and Pharmacological Neuroscience, \\ Graduate School of Biomedical Sciences, Hiroshima University, Hiroshima 734-8551, Japan
}

Cerebellar Purkinje cells (PCs) express a large amount of the $\gamma$ isoform of protein kinase C (PKC $\gamma)$ and a modest level of PKC $\alpha$. The PKC $\gamma$ is involved in the pruning of climbing fiber (CF) synapses from developing PCs, and PKC $\alpha$ plays a critical role in long-term depression (LTD) at parallel fiber (PF)-PC synapses. Moreover, the PKC signaling in PCs negatively modulates the nonselective transient receptor potential cation channel type 3 (TRPC3), the opening of which elicits slow EPSCs at PF-PC synapses. Autosomal dominant spinocerebellar ataxia type 14 (SCA14) is caused by mutations in PKC $\gamma$. To clarify the pathology of this disorder, mutant (S119P) PKC $\gamma$ tagged with GFP was lentivirally expressed in developing and mature mouse PCs in vivo, and the effects were assessed 3 weeks after the injection. Mutant PKC $\gamma$-GFP aggregated in PCs without signs of degeneration. Electrophysiology results showed impaired pruning of CF synapses from developing PCs, failure of LTD expression, and increases in slow EPSC amplitude. We also found that mutant PKC $\gamma$ colocalized with wild-type PKC $\gamma$, which suggests that mutant PKC $\gamma$ acts in a dominant-negative manner on wild-type PKC $\gamma$. In contrast, PKC $\alpha$ did not colocalize with mutant PKC $\gamma$. The membrane residence time of PKC $\alpha$ after depolarization-induced translocation, however, was significantly decreased when it was present with the mutant PKC $\gamma$ construct. These results suggest that mutant PKC $\gamma$ in PCs of SCA14 patients could differentially impair the membrane translocation kinetics of wild-type $\gamma$ and $\alpha$ PKCs, which would disrupt synapse pruning, synaptic plasticity, and synaptic transmission.

\section{Introduction}

Protein kinase C (PKC) isoforms are a family of serine/threonine kinases that play important roles in various cellular functions (Nishizuka, 1992). Among PKC subtypes, the $\gamma$ isoform of PKC (PKC $\gamma)$ is specifically expressed in the CNS and is especially abundant in cerebellar Purkinje cells (PCs) (Saito et al., 1988). In the PKC $\gamma$-deficient cerebellum, the developmental elimination of surplus climbing fiber (CF) synapses from PCs is impaired, which suggests that PKC $\gamma$ is involved in the process of pruning CF synapses from developing PCs (Kano et al., 1995).

Received Oct. 22, 2010; revised July 28, 2011; accepted Aug. 12, 2011.

Author contributions: N.S. and H. Hirai designed research; A.N.S., H. Horiuchi, T.S., G.H., T.I., and A.I. performed research; A.N.S., T.S., N.S., and H. Hirai analyzed data; H. Hirai wrote the paper.

This work was supported in part by Grant-in-Aid for Scientific Research (KAKENHI; 19670003), the Funding Program for Next Generation World-Leading Researchers (LS021), and grants from Research on Measures for Intractable Diseases (Ataxic Diseases and Neurodegenerative Diseases) from the Ministry of Health, Labor, and Welfare, the Sumitomo Foundation, the Takeda Science Foundation and the Uehara Memorial Foundation (to H. Hirai). The authors declare that they have no competing financial interests. The lentiviral vector and MSCV promoter were provided by St. Jude Children's Research Hospital and the American National Red Cross, respectively. Protein kinase C knock-out mice were provided by Dr. Masanobu Kano, University of Tokyo. We thank Dr. Nobutake Hosoi for critical reading of the manuscript.

Correspondence should be addressed to Hirokazu Hirai, Department of Neurophysiology, Gunma University Graduate School of Medicine, Maebashi, Gunma, 371-8511, Japan. E-mail: hirai@gunma-u.ac.jp.

DOI:10.1523/JNEUROSCI.5530-10.2011

Copyright $\odot 2011$ the authors $\quad 0270-6474 / 11 / 3114324-11 \$ 15.00 / 0$
Long-term depression (LTD) at parallel fiber (PF)-PC synapses (hereafter "LTD" refers to PF-PC LTD) is one form of cerebellar synaptic plasticity. Although a recent study has shown that LTD is not essential for cerebellar motor learning (Schonewille et al., 2011), the blockade of PKC activity in PCs impairs both cerebellar motor learning and LTD (De Zeeuw et al., 1998; Goossens et al., 2001; Koekkoek et al., 2003). This is presumably because PKC mediates upstream signaling, and inhibition of PKC activity disrupts not only the LTD cascade but also other signaling pathways indispensable for cerebellar motor learning.

LTD is induced by conjunctive stimulation of PF and CF, which triggers activation of the mGluR 1 subtype of metabotropic glutamate receptors, an increase in the intracellular $\mathrm{Ca}^{2+}$ concentration, and PKC activation, leading to phosphorylation of serine 880 in the carboxyl-terminal PDZ ligand motif of the AMPA receptor GluR2 (Matsuda et al., 1999, 2000; Hirai, 2001). This phosphorylation event promotes the binding of the protein interacting with protein kinase C (PICK1) with GluR2 and ultimately results in PICK1-dependent endocytosis of postsynaptic AMPA receptors (Xia et al., 2000; Hirai, 2001; Chung et al., 2003). Although PKC activation plays a critical role in cerebellar LTD expression and PKC $\gamma$ is a major isoform in PCs, cerebellar LTD was not impaired in PKC $\gamma$ knock-out mice (Chen et al., 1995). Notably, studies have suggested that $\mathrm{PKC} \alpha$, which has the potential to associate with PICK1, is the PKC isoform responsible for 
the phosphorylation of GluR2 serine 880 and the expression of cerebellar LTD (Leitges et al., 2004).

Autosomal dominant spinocerebellar ataxias (SCAs) are classified into at least 28 types by chromosomal loci of the causal genes (Carlson et al., 2009). Among these, SCA14 is caused by a missense mutation of the PRKCG gene encoding PKC $\gamma$. SCA14 patients show progressive ataxia and cerebellar atrophy accompanied by PC loss. Notably, PKC $\gamma$-deficient mice show only mild ataxia and no gross morphological abnormalities in the cerebellum (Chen et al., 1995; Kano et al., 1995), which suggests that a gain-of-toxic function, rather than a loss of function, of $\mathrm{PKC} \gamma$ underlies the pathology of SCA14. To clarify the SCA14 pathology, we expressed mutant PKC $\gamma$ with the serine at position 119 in the highly conserved membrane-binding $\mathrm{C} 1 \mathrm{~B}$ subdomain of the regulatory domain mutated to proline (S119P) in PCs in vivo, and we investigated the morphological and functional effects of this mutation. We found that mutant PKC $\gamma$ differentially interacted with wild-type PKC $\gamma$ and $\mathrm{PKC} \alpha$ and disrupted synaptic functions in PCs.

\section{Materials and Methods}

All procedures for the care and treatment of animals were carried out according to the Japanese Act on the Welfare and Management of Animals and the Guidelines for the Proper Conduct of Animal Experiments issued by the Science Council of Japan. The experimental protocol was approved by the Institutional Committee of Gunma University (07-015 and 04-44). All efforts were made to minimize suffering and reduce the number of animals used. C57BL/6 mice of both sexes were used in this study.

Lentiviral vectors and cerebellar injection. To construct the plasmids encoding wild-type or mutant (S119P) PKC $\gamma$-GFP, PKC $\gamma$ and GFP cDNAs were subcloned into the pcDNA3 expression vector (Invitrogen) as described previously (Seki et al., 2005). The GFP cDNA was subcloned $3^{\prime}$ to the PKC $\gamma$ cDNA. Wild-type or mutant PKC $\gamma$-GFP in pcDNA3 was subcloned into the pCL20c lentiviral vector plasmid, which carried the murine embryonic stem cell virus (MSCV) promoter. Vesicular stomatitis virus-G protein pseudotyped HIV vectors (Torashima et al., 2006a) were also used in this study. The backbones of helper plasmids were derived from pCAGGS (Niwa et al., 1991). The detailed procedure for viral vector production has been described in our previous reports (Torashima et al., 2006b; Iizuka et al., 2009). Viral titers were assessed by counting the number of GFP-expressing cells after transducing HEK $293 \mathrm{~T}$ cells as described previously (Torashima et al., 2006b). We used 16, 19 , and 16 batches of lentiviral vectors expressing wild-type PKC $\gamma$-GFP, mutant PKC $\gamma$-GFP, and GFP alone, respectively. The viral titers were within the range of $5 \times 10^{9}-5 \times 10^{10}$ transduction units $/ \mathrm{ml}$. The viral solution was stored at $4^{\circ} \mathrm{C}$ and used within 1 week.

The mutant PKC $\gamma$ was lentivirally expressed in the cerebella of C57BL/6 mice of two age groups: 1 week old (postnatal day (P) 6-P7) and 4 weeks old (P21-P25). Three weeks after injection, mice were anesthetized by inhalation of $2 \%$ isoflurane (the flow speed was $1 \mathrm{~L} / \mathrm{min}$ ) or an intraperitoneal injection of sodium pentobarbital $(40 \mathrm{mg} / \mathrm{kg}$ body weight), respectively, and the lentiviral solution was injected into the cerebellar cortex as described previously (Torashima et al., 2006a; Iizuka et al., 2009).

Rotarod test. The coordination and motor skill acquisition of the mice were assessed by an accelerated rotarod test. The Rota-Rod Treadmill (Muromachi Kikai) consisted of a gridded plastic rod $(3 \mathrm{~cm}$ in diameter, $10 \mathrm{~cm}$ long) flanked by two large round plates $(50 \mathrm{~cm}$ in diameter). The rod accelerated from 0 to 40 revolutions per minute (rpm) for $5 \mathrm{~min}$ and then remained at the top speed for an additional $10 \mathrm{~min}$. One test defines 4 days of 4 trials each day (a $30 \mathrm{~min}$ rest between trials), and we recorded the time that the mice spent on the rod.

Quantitative analysis of aggregates in PCs. Mice were transcardially perfused with $4 \%$ paraformaldehyde. After overnight fixation at $4{ }^{\circ} \mathrm{C}$, the vermis of the cerebellum was sagittally sliced at $50 \mu \mathrm{m}$ intervals. The slices were examined using fluorescence microscopy (DMI6000B, Leica) and confocal microscopy (LSM 5 PASCAL; Carl Zeiss). The ratio of PCs with aggregates to total PCs was determined by manually changing the focus. Purkinje cells that had GFP-positive spots only at the stem of the primary dendrites and around the nucleus were not counted as aggregate-positive cells because those GFP-positive spots were primarily included in cis-Golgi.

Western blot analysis. Cerebellar cortices that expressed wild-type or mutant PKC $\gamma$-GFP or GFP alone were examined by Western blot as described previously (Torashima et al., 2008). Blots were incubated with rabbit polyclonal anti-PKC $\gamma$ (1:200, Santa Cruz Biotechnology) and mouse monoclonal anti-GFP (1:10,000; Nacalai Tesque) antibodies. The secondary antibodies were HRP-conjugated anti-rabbit or anti-mouse IgG (Bio-Rad), respectively, at 1:4000 dilutions. Band volume on the Western blot was quantified using the Basic Quantifier (Bio Image).

Biocytin infusion and morphological analysis. For subsequent visualization of PC morphology, $0.5 \%$ biocytin (Sigma-Aldrich) diluted in intracellular solution was infused by passive diffusion through a patch pipette into whole cell-clamped PCs. Cerebellar slices were then fixed with $4 \%$ paraformaldehyde. After overnight fixation at $4^{\circ} \mathrm{C}$, slices were rinsed in 0.1 M PBS, pH 7.4 (3 times, 5 min each), and treated with PBS containing streptavidin-Alexa Fluor 594 conjugate. After a $2 \mathrm{~h}$ incubation at room temperature, the slices were mounted and coverslipped with Permount. Slices were analyzed using a confocal laser-scanning microscope (LSM 5 PASCAL). In the confocal microscopic analysis, cerebellar slices were scanned at $1 \mu \mathrm{m}$ intervals in Z-stack mode to project a whole dendritic tree onto a planar image, which was scaled according to intensity from 0 to 255 arbitrary units (AU), with 0 as the minimum intensity and 255 as the maximum. Binary images of biocytin-infused PCs were obtained by thresholding the projected images (i.e., the area brighter than 100 of 255 AU was regarded as dendrites). The dendritic area was calculated using IPLab imaging software (Scanalytics).

TUNEL staining. Fluorescent terminal deoxynucleotidyl transferase dUTP nick-end labeling (TUNEL) was performed using a kit (Roche Science). As a positive control, cerebellar sections were treated with recombinant DNase I as described in the manufacturer's directions. One hundred PCs in slices from three animals were assessed in each experimental group. TUNEL staining was also performed in cerebellar neuronal cultures using the same protocol.

Electrophysiological assay. Parasagittal cerebellar slices $(200 \mu \mathrm{m}$ in thickness) were prepared, and whole-cell recordings were conducted as described previously (Torashima et al., 2008; Iizuka et al., 2009). Briefly, mice were deeply anesthetized with inhalation of isoflurane $(3 \%)$ and killed by decapitation. The whole brain was quickly dissected out and immersed for a couple of minutes in an ice-cold solution containing the following (in mM): 234 sucrose, $26 \mathrm{NaHCO}_{3}, 2.5 \mathrm{KCl}, 1.25 \mathrm{NaH}_{2} \mathrm{PO}_{4}, 11$ glucose, $10 \mathrm{MgSO}_{4}$, and $0.5 \mathrm{CaCl}_{2} 0.5$, $\mathrm{pH} 7.4$, when bubbled with $95 \%$ $\mathrm{O}_{2}$ and $5 \% \mathrm{CO}_{2}$ ). Parasagittal slices of cerebellar vermis were obtained using a microslicer (ZERO1; Dosaka EM). The slices were maintained in an extracellular solution containing the following (in $\mathrm{mM}$ ): $125 \mathrm{NaCl}, 2.5$ $\mathrm{KCl}, 2 \mathrm{CaCl}_{2}, 1 \mathrm{MgCl}_{2}, 1.25 \mathrm{NaH}_{2} \mathrm{PO}_{4}, 26 \mathrm{NaHCO}_{3}, 10$ D-glucose, and 0.1 picrotoxin. This solution was bubbled continuously with a mixture of $95 \% \mathrm{O}_{2}$ and $5 \% \mathrm{CO}_{2}$ at room temperature for at least $45 \mathrm{~min}$ before beginning the recordings. Purkinje cells were voltage-clamped at -70 $\mathrm{mV}$ to record PF EPSCs and $-10 \mathrm{mV}$ to record CF EPSCs. Selective stimulation of CFs and PFs was confirmed by paired-pulse depression and paired-pulse facilitation of EPSC amplitudes, respectively, with a 50 $\mathrm{ms}$ interstimulus interval.

Long-term depression was examined in PCs from homozygous and heterozygous PKC $\gamma$-knock-out mice (P30-P31), as well as PCs expressing wild-type or mutant PKC $\gamma$-GFP or GFP alone. The electrical stimulation protocol for LTD induction was described in our previous reports (Hirai et al., 2005; Torashima et al., 2008). Briefly, PF EPSCs were successively recorded from PCs voltage-clamped at $-70 \mathrm{mV}$ and a frequency of $0.1 \mathrm{~Hz}$. We applied conjunctive stimulation that consisted of 30 single PF stimuli at $1 \mathrm{~Hz}$ together with a $200 \mathrm{~ms}$ depolarizing pulse from a holding potential of -70 to $+20 \mathrm{mV}$. The averaged amplitudes of PF EPSCs over 1 min were normalized to the baseline value, which was the average of the $1 \mathrm{~min}$ responses (six traces) that occurred just before conjunctive stimulation. 
For recordings of mGluR1-mediated slow EPSCs, the strength of the electrical stimulation was initially adjusted to produce AMPA receptor-mediated fast EPSCs with an amplitude of $500 \mathrm{pA}$. We subsequently applied 6-cyano-2, 3-dihydroxy-7-nitro-quinoxaline (CNQX), an AMPA-type glutamate receptor antagonist, and slow EPSCs were elicited by applying 10 or 25 electrical stimuli to PFs at $200 \mathrm{~Hz}$.

Adenoviral vectors. To express wild-type $\mathrm{PKC} \gamma$-DsRed-monomer (DRm) or PKC $\alpha$ DRm with mutant PKC $\gamma$-GFP in cultured PCs, we constructed two types of adenoviral vectors as described previously (Seki et al., 2009). The first vector, Ad-L7-tTA, expressed tetracycline (tet) transactivator (tTA) under control of the PC-specific L7 promoter. The second set of vectors, Ad-TetOP-WTPKC $\gamma$-DRm, AdTetOP-WTPKC $\alpha$-DRm, and Ad-TetOP$\mathrm{MuPKC} \gamma$-GFP, expressed respective genes under control of a tet-responsive promoter that is transactivated by tTA. Viral vectors were produced using an AdEasy adenoviral vector system (Stratagene).

Expression and live imaging of $P K C \gamma$-GFP in cultured PCs. Cerebellar dissociated cultures were prepared as described previously (Seki et al., 2009). On days in vitro (DIV) 14-21, cultured cells were infected with two adenoviral vectors, i.e., Ad-L7-tTA $\left(4 \times 10^{6}\right.$ plaque-forming U/dish) and Ad-TetOP-WT PKC $\gamma$-DRm, Ad-TetOP-WTPKC $\alpha$-DRm, or Ad-TetOP-MuPKC $\gamma$-GFP $\left(\sim 6 \times 10^{5}\right.$ plaque-forming units/dish). After $7 \mathrm{~d}$ of cultivation, GFP fluorescence and DRm fluorescence were detected with $488 \mathrm{~nm}$ argon laser excitation using a $505-530 \mathrm{~nm}$ bandpass barrier filter and $543 \mathrm{~nm}$ helium/neon laser excitation using a 560 nm long-pass barrier filter, respectively, under a confocal laser scanning fluorescent microscope (LSM 510 META; Carl Zeiss). Purkinje cells were morphologically characterized by relatively large somata and highly branched dendrites. PKC $\gamma$-GFP fluorescence images of PCs were obtained as Z-stack images.

Observation of PKC $\gamma-G F P$ and PKC $\alpha-D R m$ translocation. On DIV 2128, the culture medium was replaced with $950 \mu \mathrm{l}$ of HEPES buffer (in mм: $165 \mathrm{NaCl}, 5 \mathrm{KCl}, 1 \mathrm{CaCl}_{2}, 1 \mathrm{MgCl}_{2}, 5$ HEPES, 10 glucose, $\mathrm{pH}$ 7.4). Translocation of mutant PKC $\gamma$-GFP, wild-type PKC $\gamma$-DRm, and PKC $\alpha$ DRm was triggered by direct application of $50 \mu \mathrm{l}$ of HEPES buffer containing a high concentration of $\mathrm{KCl}(100 \mathrm{mM})$. Green fluorescent protein and DRm fluorescent images of PC dendrites were recorded every $0.5 \mathrm{~s}$ for $2 \mathrm{~min}$ before and after stimulation using a confocal laser microscope. The half-time of fluorescence recovery was calculated by fitting temporal changes in fluorescence intensity after the maximal reduction of cytosolic fluorescence to a one-site binding curve model using Prism 4 software (GraphPad Software). All experiments were performed at $37^{\circ} \mathrm{C}$.

Statistical analysis. The values obtained are expressed as the means \pm SEM unless otherwise specified, and $n$ indicates the number of tested Purkinje cells. Statistical analyses of differences between groups were performed using the Kruskal-Wallis test, unpaired $t$ test, or one-way ANOVA followed by Tukey's post hoc test. A probability of 0.05 was taken as the level of statistical significance.

\section{Results}

All PKC $\gamma$ mutants found in SCA14 patients were susceptible to the formation of aggregates when heterologously expressed in Chinese hamster ovary cells (Seki et al., 2005). In addition, new $\mathrm{PKC} \gamma$ mutants identified after the experiments described in this report also possessed similar aggregate-prone properties in culture preparations (T. Seki and N. Sakai, unpublished observation). Thus, aggregation seems to be a hallmark of all PKC $\gamma$ mutants identified in SCA14 patients. Among PKC $\gamma$ mutations,
Injected at P6-P7

B

Injected at P21-P25
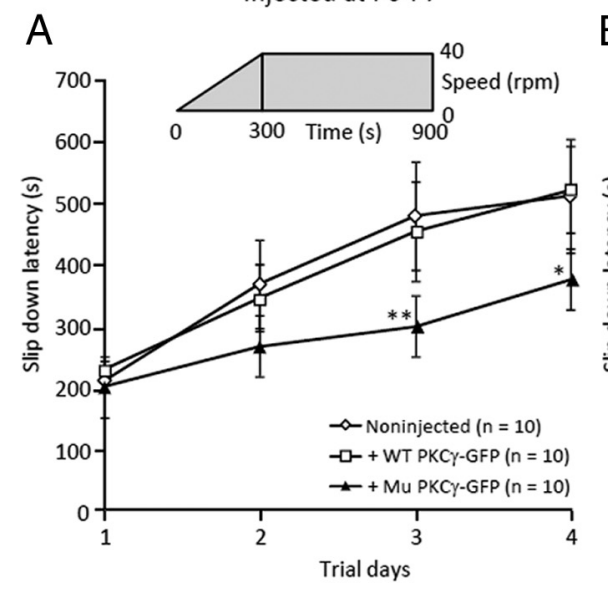
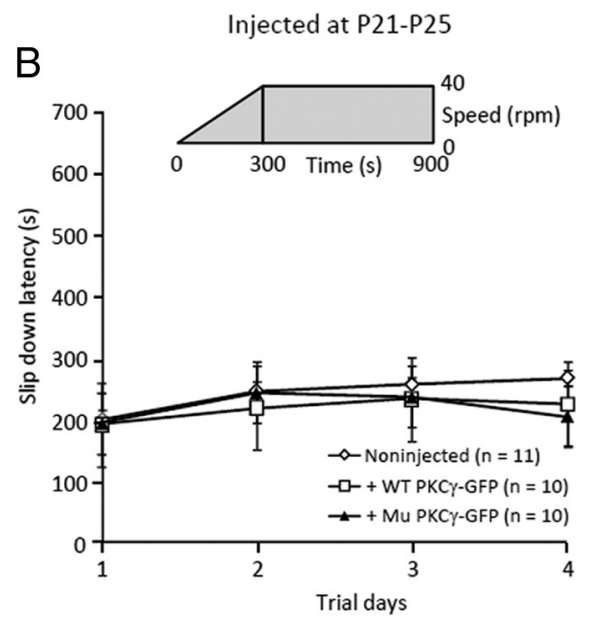

Trial days

Figure 1. Rotarod performance of mice lentivirally treated at 1 week of age or 4 weeks of age. As depicted in the graphs, $\sim$ Noninjected $(n=10)$ $-\square+$ WT PKC $\gamma$-GFP $(n=10)$ $\neg+$ Mu PKC $\gamma-$ GFP $(n=10)$ the rod accelerated from 0 to $40 \mathrm{rpm}$ in $5 \mathrm{~min}$ and remained at $40 \mathrm{rpm}$ for an additional $10 \mathrm{~min}$. Three weeks after the injection, mice were trained in four trials per day for 4 days. Naive littermates were used as controls in each group. $A$, We observed impaired performance in mice treated at 1 week of age with lentiviral vectors expressing mutant (Mu) PKC $\gamma$-GFP weeks of age. The number of mice tested is shown in parentheses. Asterisks indicate a statistically significant difference compared with noninjected mice, which was determined by one-way ANOVA followed by Tukey's $p<0.01$.

the $1119 \mathrm{P}$ mutant is the most susceptible to aggregate formation, and the pathological significance of the aggregates has been well studied using the S119P mutant expressed in cultured cells (Seki et al., 2007, 2009, 2010; Yamamoto et al., 2010). Therefore, we focused on the S119P mutant in the present in vivo study.

\section{Assessment of coordination and motor skill acquisition by rotarod}

We injected lentiviral vectors expressing mutant or wild-type PKC $\gamma$-GFP into developing cerebella (one-week-old mice) or mature cerebella (4-week-old mice). The former was useful to assess the influence of mutant PKC $\gamma$ expression on cerebellar development, and mice were analyzed after overall maturation of the cerebellar neural circuit (P28, 3 weeks after injection). The latter was used to examine the effects of the mutant PKC $\gamma$ on PCs after establishment of the cortical circuit. Although we aimed to assess the primary effects of mutant PKC $\gamma$ expression, we also waited for 3 weeks in this age group because mutant PKC $\gamma$ is expressed more slowly in mature mice than in developing pups, and 3 weeks was needed for sufficient accumulation of mutant $\mathrm{PKC} \gamma$ in PCs. Lentivirally treated and nontreated mice were tested on the accelerating rotarod apparatus. Although lentivirally treated mice had no overt gait ataxia, One-week-old mice that had been treated with lentiviral vectors expressing mutant PKC $\gamma$-GFP exhibited significantly decreased times on the rotarod than age-matched noninjected littermates $(p<0.01$ at day 3 and $p<0.05$ at day 4) (Fig. $1 A$ ). Notably, we could not detect a significant difference in rotarod performance between mice that received the lentiviral vector injection at 4 weeks of age and agematched noninjected littermates (Fig. $1 B$ ).

After the rotarod test, we examined the transduced area in the cerebellum and the localization of GFP-fused PKC $\gamma$ in PCs. One problem of using viral vectors for the generation of disease model animals is the variation in the levels and area of transgene expression, which depends on the injection technique and the size of the target region. We found that transduction was limited to a couple of cerebellar lobules in mice treated at 4 weeks of age, whereas a much wider area of the cerebellum was transduced when lentivi- 


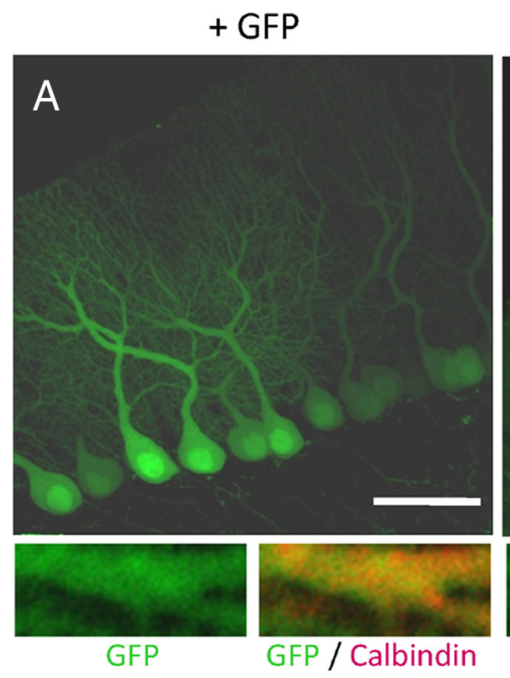

$\mathrm{D}$

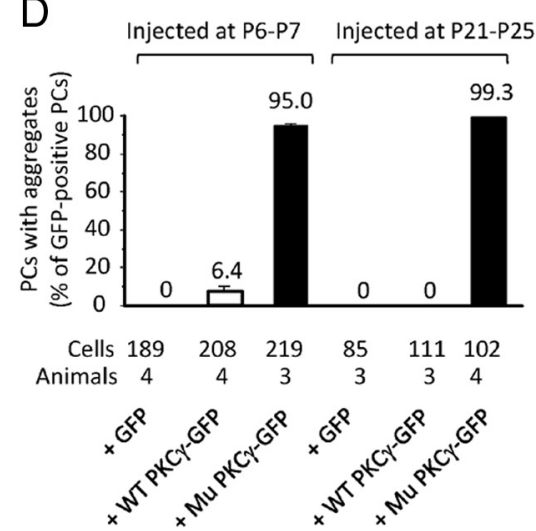

+ WT PKC $\gamma$-GFP

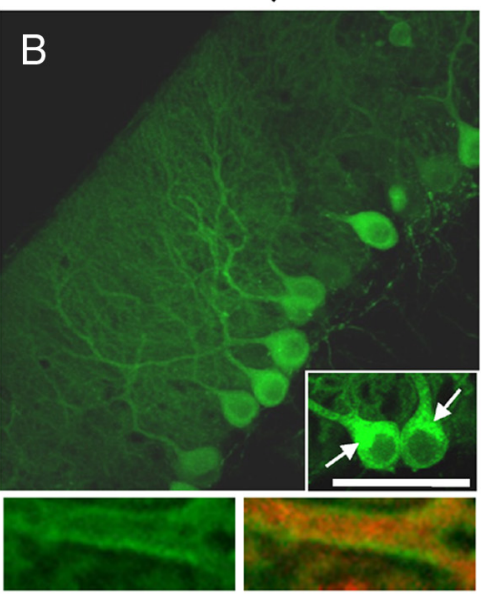

+ Mu PKC $\gamma$-GFP

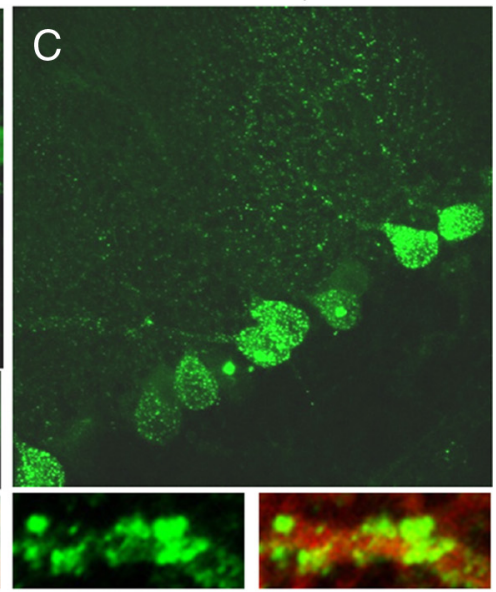

E

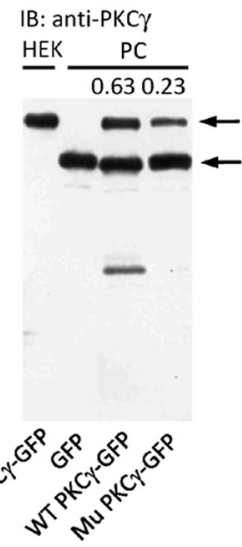

F IB: anti-GFP

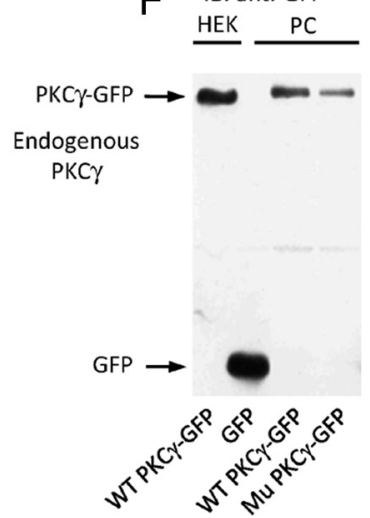

Figure 2. Aggregate formation in PCs expressing mutant PKC $\gamma$-GFP. $A-C$, GFP fluorescence images of PCs from mice treated at 1 week of age with lentiviral vectors expressing GFP (A), wild-type (WT) PKC $\gamma$-GFP (B), or mutant (Mu) PKC $\gamma$-GFP (C). Arrows in the inset of $\boldsymbol{B}$ show wild-type PKC $\gamma$-GFP in cis-Golgi. Lower small panels are enlarged images of PC dendrites, and the left and right images show native GFP fluorescence and GFP plus immunofluorescence for calbindin, respectively. Scale bars, $50 \mu \mathrm{m}$. $D$, Ratios of PCs with aggregates to total PCs from mice that received an injection of lentiviral vectors expressing GFP, wild-type PKC $\gamma$-GFP, or mutant PKC $\gamma$-GFP at 1 week of age or 4 weeks of age. The number of cells and animals examined are presented below the graph. $\boldsymbol{E}, \boldsymbol{F}$, Western blot results showing the amount of wild-type and mutant PKC $\gamma$-GFP and endogenous PKC $\gamma$ in PCs. GFP or wild-type or mutant PKC $\gamma$-GFP was lentivirally expressed in PCs in vivo before Western blot analysis. The membrane was immunoblotted (IB) with anti-PKC $\gamma(\boldsymbol{E})$ and anti-GFP $(\boldsymbol{F})$ antibodies. Numbers above the right two lanes in $(\boldsymbol{E})$ indicate ratios of wild-type or mutant $\mathrm{PKC} \gamma(-\mathrm{GFP})$ to endogenous PKC $\gamma$; the ratios of the band volume were quantified from enhanced chemiluminescence.

ral vectors were injected into the cerebella of 1-week-old pups (data not shown). This may explain why we did not observe a significant deterioration of rotarod performance in 4-week-old mice that received the lentiviral vector.

\section{Aggregation of mutant PKC $\boldsymbol{\gamma}$-GFP in PCs in vivo}

Fluorescence was observed almost homogeneously in the soma and dendrites of PCs expressing only GFP (Fig. $2 A, B$ and supplemental Fig. $1 A$, available at www.jneurosci.org as supplemental material), whereas a robust fluorescence from wild-type PKC $\gamma$ GFP was detected in the somatic region around the nucleus and stem of the primary dendrite (Fig. $2 B$ and supplemental Fig. $1 C$, arrows). These GFP-positive spots were immunolabeled for GM130, a marker for cis-Golgi (data not shown), and positive staining for GM130 suggested that those spots were localized in cis-Golgi and were different from pathological aggregates. In the dendrites, wild-type PKC $\gamma$-GFP was localized along the plasma membrane (bottom of Fig. $2 B$ and supplemental Fig. $1 C, D$ ), which was similar to the distribution of endogenous PKC $\gamma$ (supplemental Fig. $1 B$ ). In contrast, numerous aggregates of mutant PKC $\gamma$-GFP were detected in the soma and dendritic tree (Fig. $2 C$ and supplemental Fig. $1 E$ ), and aggregates in the dendrites were primarily observed at the branch points. Quantitative analysis revealed that $0-6 \%$ of PCs expressing wild-type PKC $\gamma$-GFP formed aggregates, whereas $>95 \%$ of PCs expressing mutant PKC $\gamma$-GFP exhibited aggregation regardless of the injection stage (Fig. 2D).

The aggregates may have been caused by extremely high expression levels of mutant PKC $\gamma$ protein. To test this hypothesis, expression levels of mutant PKC $\gamma$-GFP were compared with those of endogenous PKC $\gamma$ by Western blot analysis. Lentiviral vector-injected cerebellar slices were observed under a fluorescence stereomicroscope, and only GFP-expressing regions were excised for the analysis. Quantification of the band area and intensity in the Western blots revealed that the amount of recombinant wild-type and mutant PKC $\gamma$-GFP virally expressed in PCs was 63 and 23\%, respectively, of the endogenous PKC $\gamma$ level (Fig. $2 E, F)$. Compared with the wild-type construct, lower levels of mutant PKC $\gamma$-GFP in PCs probably resulted from the mutant protein being degraded by cellular quality control machinery (Yamamoto et al., 2010). Thus, in vivo aggregate formation in PCs was caused by the mutation rather than by excessive expression of mutant PKC $\gamma$-GFP. 
Normal dendritic differentiation and lack of apoptosis in PCs expressing mutant PKC $\gamma$-GFP

Previous studies in cerebellar neuronal cultures have shown that the dendrite extension of developing PCs expressing mutant (S119P) PKC $\gamma$-GFP was significantly impaired compared with PCs expressing wild-type PKC $\gamma$-GFP (Seki et al., 2009). In the present study, we examined whether similar morphological changes were present in developing PCs in vivo. The dendritic areas of PCs from noninjected mice and those infected with lentiviral vectors at $\mathrm{P} 6-\mathrm{P} 7$ were measured at the fourth postnatal week, but there was no significant difference in dendritic area between control PCs and those expressing wild-type or mutant PKC $\gamma$-GFP (supplemental Fig. 2, available at www.jneurosci. org as supplemental material).

A previous study used nuclear fragmentation analysis to show a higher rate of apoptosis in PCs having mutant $\mathrm{PKC} \gamma$ GFP aggregates compared with PCs expressing wild-type PKC $\gamma$-GFP (Seki et al., 2009). We tested whether this was also true in PCs in vivo using TUNEL staining. Although PCs in mutant PKC $\gamma$ GFP-expressing cerebella had numerous aggregates, we did not detect any TUNELpositive apoptotic PCs (supplemental Fig. 3 , available at www.jneurosci.org as supplemental material). Notably, this result cannot be attributed to methodological differences in the detection of apoptosis, because the same TUNEL staining protocol consistently labeled the apoptotic PCs that had aggregates in culture (supplemental Fig. 4, available at www.jneurosci.org as supplemental material). The inconsistency between culture and in vivo preparations may be due to the deficiency of trophic factors, such as brain-derived neurotrophic factor and glial-derived neurotrophic factor, in the culture environment, which has been observed in PKC knockout mice (Kano et al., 1995; Schrenk et al., 2002). Indeed, chronic deficiency of trophic factors and secondary defects may be required to induce apoptosis of PCs in vivo.

\section{Failure to remove surplus CFs from PCs expressing mutant PKC $\gamma$-GFP}

Immature rodent PCs form synapses with multiple CFs. As they grow, redundant CF synapses are removed, and a one PC-to-one CF relationship is established by the end of the third postnatal week (Kano and Hashimoto, 2009). A previous PKC $\gamma$ knock-out study showed the critical involvement of PKC $\gamma$ in the developmental elimination of surplus CFs from PCs (i.e., one-third of PKC $\gamma$-lacking PCs are persistently innervated by multiple CFs) (Kano et al., 1995). Using a slice patch-clamp technique, we examined whether CF pruning was properly taking place in PCs (>P21) expressing mutant PKC $\gamma$-GFP. Whole-cell patch-clamp analysis was performed in PCs lentivirally infected at P6-P7 or
P21-P25. Lentiviral vector infection and subsequent expression of GFP alone or wild-type or mutant PKC $\gamma$-GFP in PCs did not influence the access resistance, passive membrane properties [e.g., membrane capacitance $\left(C_{\mathrm{m}}\right)$ and membrane resistance $\left(R_{\mathrm{m}}\right)$ ], kinetics of PF- and CF-mediated EPSCs (supplemental Table 1, available at www.jneurosci.org as supplemental material), averaged input-output relationship of PF EPSCs (supplemental Fig. 5A, $B$, available at www.jneurosci.org as supplemental material), or short-term synaptic plasticity in response to paired pulse stimulation of PFs (supplemental Fig. 5C,D) or CFs (supplemental Fig. 5E,F). The number of CFs innervating a single PC was assessed by counting the number of CF EPSC steps that were elicited by gradually increasing the electrical stimulation to CFs. We found that about one-third of 4-week-old PCs expressing mutant PKC $\gamma$-GFP (P6-P7 viral injection) were innervated by multiple CFs (Fig. $3 A, C$ ), and this ratio was almost identical to the ratio observed in PKC $\gamma$-deficient PCs (Kano et al., 1995). After completion of the one PC-to-one $\mathrm{CF}$ relationship (i.e., mice injected at P21-P25), expression of mutant PKC $\gamma$-GFP in PCs did not influence the ratio of PCs with multiple CF innervations 

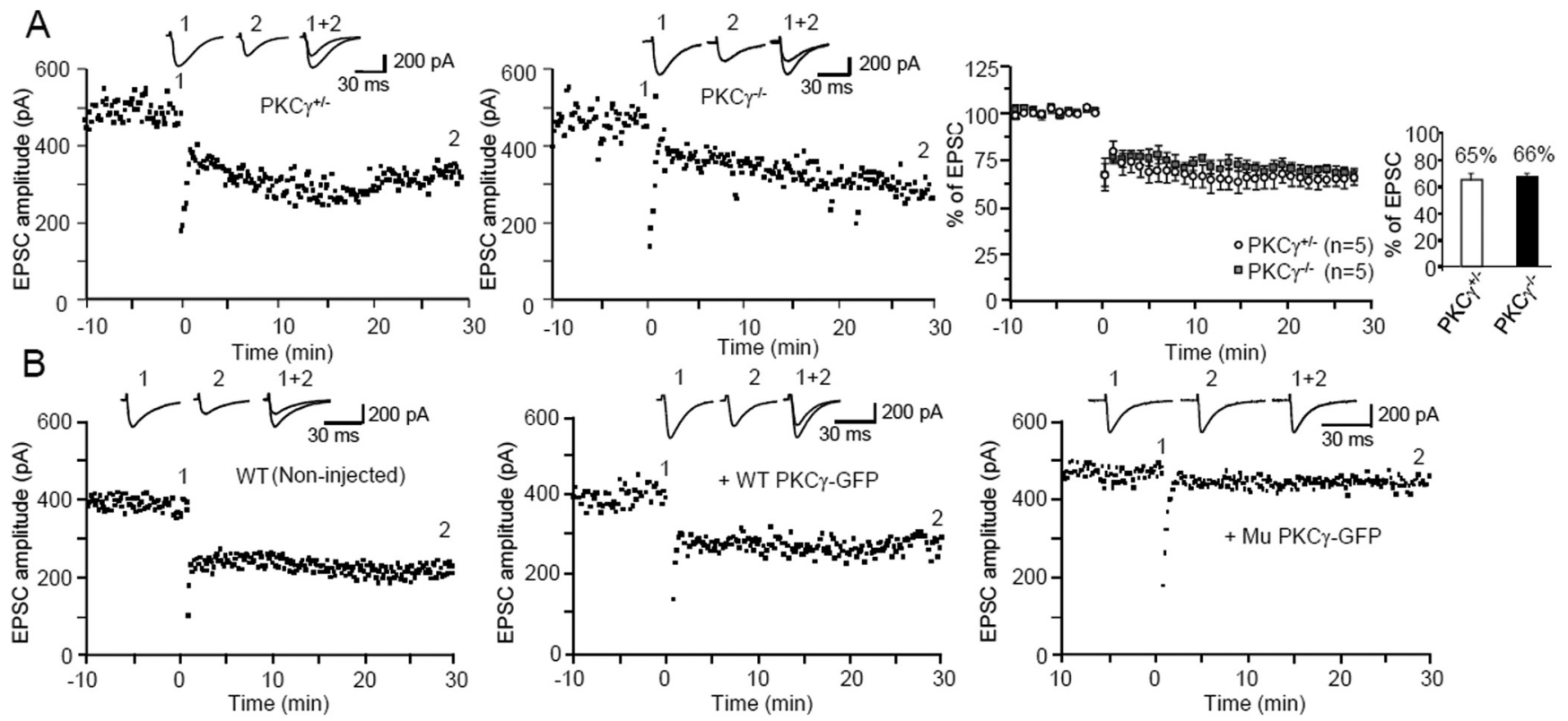

C
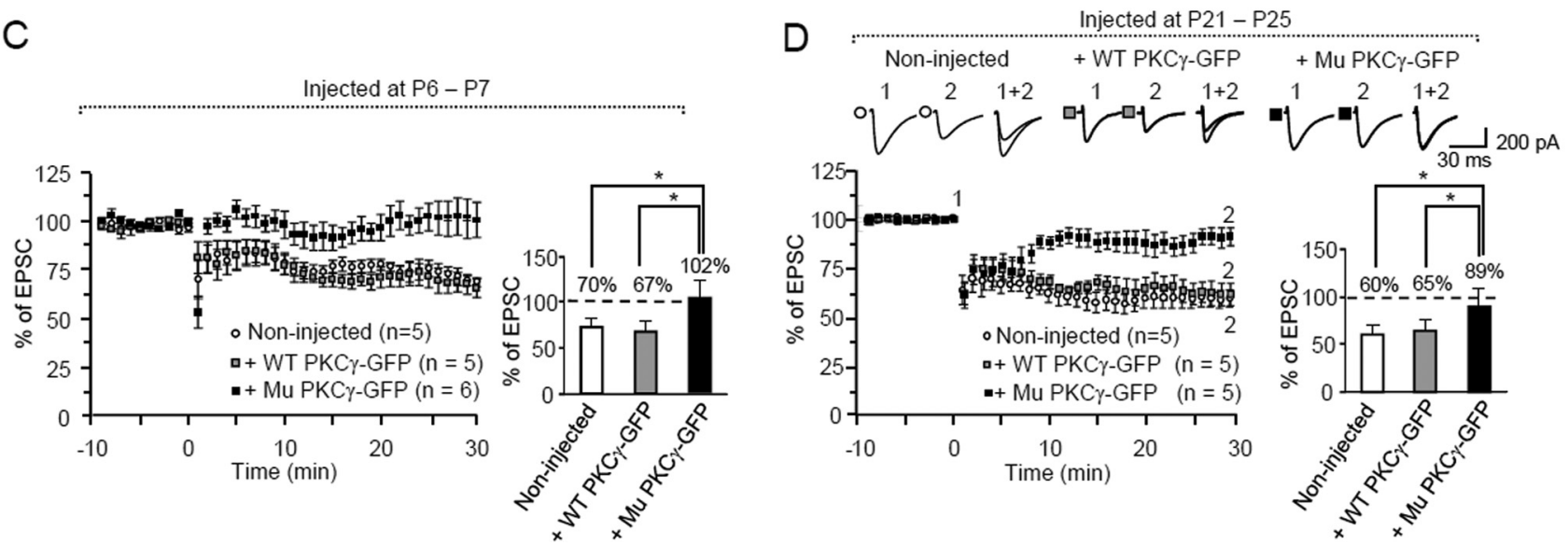

Figure 4. Deficient cerebellar LTD in PCs expressing mutant PKC $\gamma$-GFP. Conjunctive stimulation of PF $(1 \mathrm{~Hz}, 30$ s) with depolarization was applied at time $0 . A$, Expression of LTD in PKC $\gamma$-deficient PCs. Left and middle graphs show representative time course of changes in PF EPSC amplitude after conjunctive stimulation. PF EPSCs were recorded in P30-P31 PCs from heterozygous (PKC $\gamma^{+/-}$) (left) and homozygous (PKC $\gamma^{-I-}$ ) (middle) PKC $\gamma$-knock-out mice. Right graph depicts summary of the time course of changes in PF EPSC amplitude in PCs from heterozygous and homozygous PKC $\gamma$-knock-out mice. The bar graph next to the averaged graph shows the percentage of the EPSC amplitude 30 min after conjunctive stimulation relative to the baseline value. $\boldsymbol{B}$, Representative examples of changes in PF EPSC amplitude after conjunctive stimulation. PF EPSCs were recorded in P28 PCs from noninjected controls (left) and age-matched PCs expressing wild-type PKC $\gamma$-GFP (middle) or mutant PKC $\gamma$-GFP (right) from wild-type mice injected at P6-P7. C, D, Summary graphs showing the time course of changes in PF EPSC amplitude (left) and the percentage of the EPSC amplitude 30 min after conjunctive stimulation relative to the baseline value (right). PF EPSCs were recorded in PCs from noninjected mice or PCs expressing wild-type (WT) or mutant (Mu) PKC $\gamma$-GFP from mice that received a lentiviral injection at P6-P7 ( () or P21-P25 (D). In summarized graphs $(\boldsymbol{A}, \boldsymbol{C}, \boldsymbol{D})$, the averaged amplitudes of PF EPSC over 1 min were normalized to the baseline value, which was the average of the 1 min responses (six traces) that occurred just before conjunctive stimulation. The numbers $(n)$ of tested $P C s$ and animals (number of tested $P C s$ equals the number of animals examined) are indicated in the graph. Insets 1,2 , and $1+2$ in $\boldsymbol{A}, \boldsymbol{B}$ and $\boldsymbol{D}$ show representative traces of PF EPSC just before conjunctive stimulation (1) and 30 min after conjunctive stimulation (2), as well as a superimposed image $(1+2)$. Asterisks indicate statistically significant differences determined by one-way ANOVA followed by Tukey's post hoc test; ${ }^{*} p<0.05$.

(Fig. $3 B, D$ ). There were no significant differences in the ratio of PCs with multiple CF innervations between PCs from noninjected mice and those virally expressing wild-type PKC $\gamma$-GFP or GFP alone (Fig. 3C,D).

\section{Failure of LTD expression in PCs expressing} mutant PKC $\gamma$-GFP

A previous study revealed robust LTD expression in PKC $\gamma$ deficient PCs (Chen et al., 1995). Thus, we examined whether LTD was induced in PCs expressing mutant PKC $\gamma$-GFP. Because our LTD induction protocol (30 single PF stimuli at $1 \mathrm{~Hz}$ together with a $200 \mathrm{~ms}$ depolarizing pulse) was quite different from the protocol used in the studies with PKC $\gamma$-knock-out mice (Chen et al., 1995), we verified whether LTD could be reliably induced by our protocol in PCs from PKC $\gamma$-knock-out mice. Similar to the results in $\mathrm{PKC} \gamma^{+/-} \mathrm{PCs}$, our stimulation protocol induced robust LTD in PKC $\gamma^{-1-}$ PCs (Fig. 4A). Notably, although our protocol induced LTD in wild-type PKC $\gamma$-GFP-expressing PCs from mice lentivirally treated at 1 week of age, the same stimuli failed to induce LTD in mutant PKC $\gamma$-GFP-expressing PCs (Fig. $4 B, C)$. In addition, LTD was not induced in mutant PKC $\gamma$-GFPexpressing PCs lentivirally treated at the fourth postnatal week (Fig. 4D). Conjunctive stimulation decreased the PF EPSC amplitude to $60-65 \%$ of the prestimulation levels in naive PCs and in those expressing wild-type PKC $\gamma$-GFP, whereas it decreased the PF-EPSC amplitude to only $89 \%$ in mutant PKC $\gamma$-GFPexpressing PCs. 


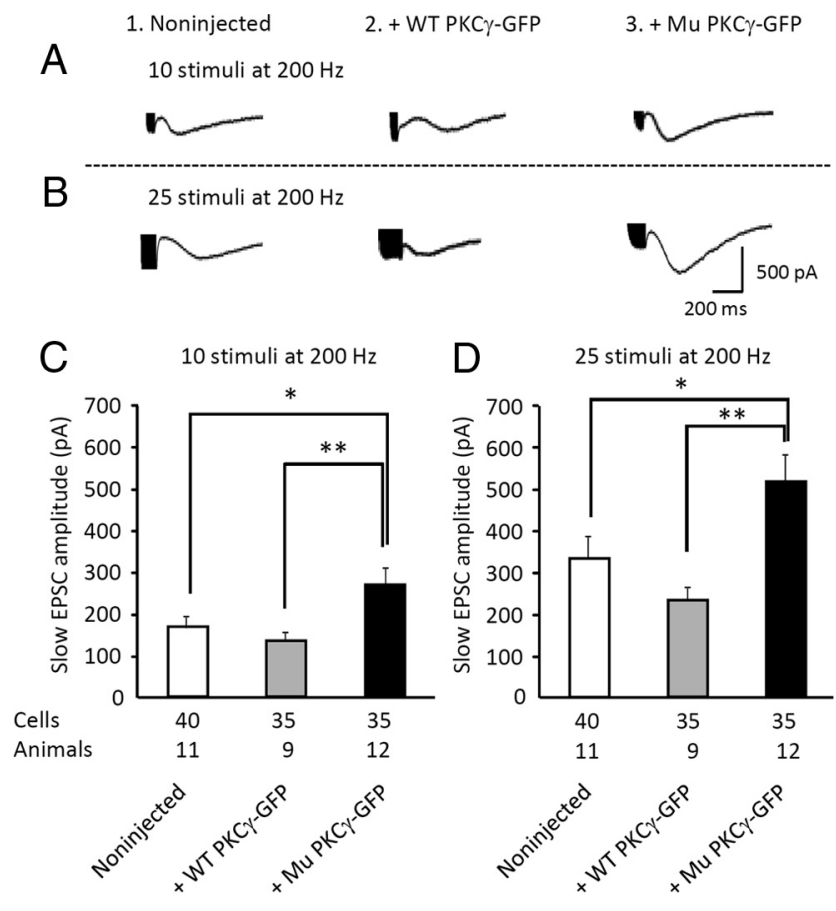

Figure 5. Increase in the amplitude of mGluR1-mediated slow EPSCs by mutant PKC $\gamma$ GFP expression. $\boldsymbol{A}, \boldsymbol{B}$, Representative mGluR1-mediated slow EPSCs elicited in naive PCS (1) or PCs expressing wild-type (WT) PKC $\gamma$-GFP (2) or mutant (Mu) PKC $\gamma$-GFP (3). Slow EPSCS were evoked by electrical stimulation of PFs with 10 stimuli $(\boldsymbol{A})$ or 25 stimuli $(\boldsymbol{B})$ at $200 \mathrm{~Hz}$. The strength of electrical stimulation was adjusted before application of CNQX, an AMPA receptor antagonist, to obtain an amplitude of $500 \mathrm{pA}$ for AMPA receptor-mediated EPSCs. C, D Summary graphs showing the amplitude of slow EPSCs from naive PCs or PCs expressing wild-type PKC $\gamma$-GFP or mutant PKC $\gamma$-GFP upon electrical stimulation of PFs with 10 stimuli $(\boldsymbol{C})$ or 25 stimuli $(\boldsymbol{D})$ at $200 \mathrm{~Hz}$. The numbers of cells and animals examined are presented just below the graph. Asterisks indicate statistically significant differences compared with noninjected mice as determined by one-way ANOVA followed by Tukey's post hoc test; ${ }^{*} p<0.05,{ }^{* *} p<0.01$.

\section{Slow EPSC amplitude is increased by mutant PKC $\gamma$-GFP expression}

Activation of mGluR1 causes the opening of a nonselective transient receptor potential cation channel type 3 (TRPC3) in PCs (Hartmann et al., 2008), which can be electrophysiologically recorded as a slow EPSC. Recently, studies have reported that TRPC3 is a substrate of PKC $\gamma$ and that wild-type PKC $\gamma$, but not SCA14 mutant PKC $\gamma($ G128D), negatively regulates TRPC3 activity in COS-7 cells (Adachi et al., 2008). Thus, we examined whether mutant PKC $\gamma$-GFP expression modulated mGluR1mediated slow EPSCs in PCs from mice that received a viral injection at P21-P25. The amplitudes of slow EPSCs evoked by 10 stimuli at $200 \mathrm{~Hz}$ were $166.0 \pm 27.4 \mathrm{pA}(n=40 \mathrm{PCs}$ from 11 noninjected mice), $133.6 \pm 20.3 \mathrm{pA}$ ( $n=35$ wild-type PKC $\gamma$ expressing PCs from 9 mice), and $268.7 \pm 41.2 \mathrm{pA}(n=35$ mutant PKC $\gamma$-GFP-expressing PCs from 12 mice) (Fig. 5A, C). Increasing the number of PF stimulations ( 25 stimuli at $200 \mathrm{~Hz}$ ) produced larger slow EPSCs in noninjected PCs (331.3 \pm 53.2 pA, $n=40$ cells from 11 mice) and PCs expressing wild-type (232.1 $\pm 30.2 \mathrm{pA}, n=30$ cells from 9 mice) or mutant $(514.5 \pm$ $53.4 \mathrm{pA}, n=35$ cells from 12 mice) PKC $\gamma$-GFP (Fig. $5 B, D$ ). In both stimulation protocols, mutant PKC $\gamma$-GFP expression significantly increased the amplitude of slow EPSCs $(p<0.05$, Tukey's post hoc test after one-way ANOVA), which suggests that mutant PKC $\gamma$-GFP expression canceled the inhibitory effect of endogenous PKC $\gamma$. In contrast, increasing PKC $\gamma$ levels by ex- pressing wild-type PKC $\gamma$-GFP did not significantly alter the slow EPSC amplitude. We hypothesized that the PKC-mediated inhibitory action on slow EPSCs may be saturated by endogenous levels of PKC $\gamma$.

\section{No influence of mutant PKC $\gamma$-GFP on endocannabinoid- mediated presynaptic inhibition}

A series of recent studies revealed that a brief burst of PF stimulation in PCs caused mGluRl activation and local $\mathrm{Ca}^{2+}$ elevation due to AMPA receptor-mediated depolarization. In addition, these effects produce endocannabinoids, which leads to endocannabinoid-mediated retrograde suppression of glutamate release from PF terminals (Maejima et al., 2001; Brown et al., 2003; Marcaggi and Attwell, 2005, 2007). Endocannabinoid signaling has been hypothesized to be required for LTD expression, because LTD is blocked by an antagonist of endocannabinoid CB1 receptors (Safo and Regehr, 2005). In addition, LTD was deficient in CB1 knock-out mice (Safo and Regehr, 2005). Therefore, we examined whether mutant PKC $\gamma$-GFP affected the endocannabinoid-mediated retrograde suppression of synaptic transmission at PF-PC synapses. Tetanic stimulation of PFs with 150 stimuli at $300 \mathrm{~Hz}$ decreased PF EPSC amplitude in PCs expressing mutant PKC $\gamma$ GFP to a similar level as that in the control PCs (supplemental Fig. 6, available at www.jneurosci.org as supplemental material). Notably, the decrease in PF EPSC amplitude was completely reversed by the addition of the $\mathrm{CB} 1$ receptor inhibitor AM 251, which suggests that the observed suppression of PF EPSCs was mediated by the endocannabinoid-CB1 receptor pathway and that mutant PKC $\gamma$-GFP expression in PCs had no significant influence on this short-term synaptic plasticity.

\section{Coaggregation of mutant PKC $\gamma$ with wild-type PKC $\gamma$ but not PKCa}

Although mutant PKC $\gamma$-GFP-expressing PCs had a sufficient amount of endogenous PKC $\gamma$, we observed that developmental pruning of CFs from PCs was impaired (Fig. 2E), which suggests that mutant PKC $\gamma$-GFP works in a dominant-negative manner. To clarify the detailed mechanism, wild-type PKC $\gamma$-DRm was simultaneously expressed with mutant PKC $\gamma$-GFP in cultured PCs. We found that wild-type PKC $\gamma$-DRm formed robust coaggregates with mutant PKC $\gamma$-GFP (Fig. 6A-C). This result strongly suggests that mutant $\mathrm{PKC} \gamma$-GFP disrupts endogenous $\mathrm{PKC} \gamma$ function by coaggregation, which could account for the disruption of CF pruning in developing PCs.

Because LTD expression, which is mediated by $\operatorname{PKC} \alpha$, was impaired in PCs expressing mutant PKC $\gamma$-GFP, we hypothesized that $\mathrm{PKC} \alpha$ coaggregated with mutant PKC $\gamma$-GFP in a manner similar to that of wild-type PKC $\gamma$. To test this possibility, PKC $\alpha$ DRm was coexpressed with mutant PKC $\gamma$-GFP in cultured PCs. Notably, PKC $\alpha$-DRm was diffusely distributed in the soma and dendrites and did not coaggregate with mutant PKC $\gamma$ GFP (Fig. 6D-I).

\section{Membrane residence time of PKC $\alpha$ was shortened by mutant PKC $\gamma$}

Previous in vitro studies have shown that $\mathrm{C} 1 \mathrm{~B}$ domain mutants of PKC $\gamma$ have decreased affinity for membrane lipids (Sakai et al., 1997). Although these mutants translocated to the plasma membrane in response to phorbol ester stimulation, they had significantly shorter residence times at the membrane than wild-type PKC $\gamma$ (Adachi et al., 2008). Notably, alterations in the translocation and/or membrane residence time of PKC $\alpha$ in PCs expressing 


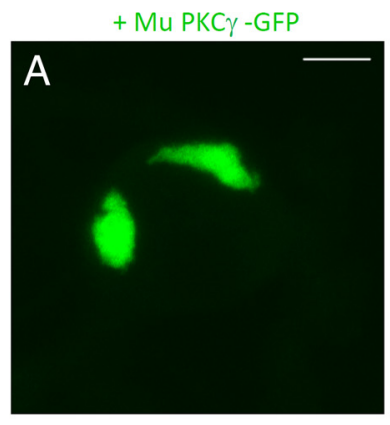

$+\mathrm{Mu}$ PKC $\gamma-\mathrm{GFP}$

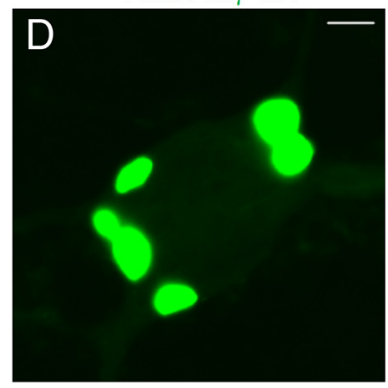

$+\mathrm{Mu} P K C \gamma-G F P$

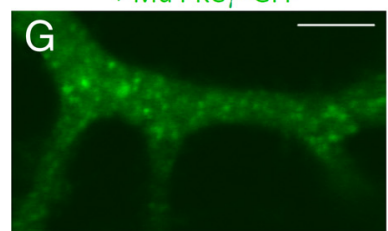

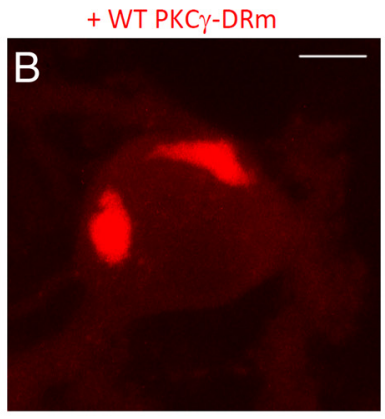

+WT PKC $\alpha-D R m$

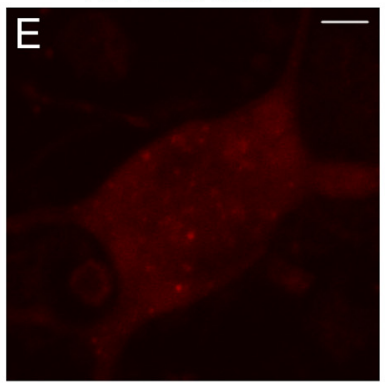

+WT PKC $\alpha-D R m$
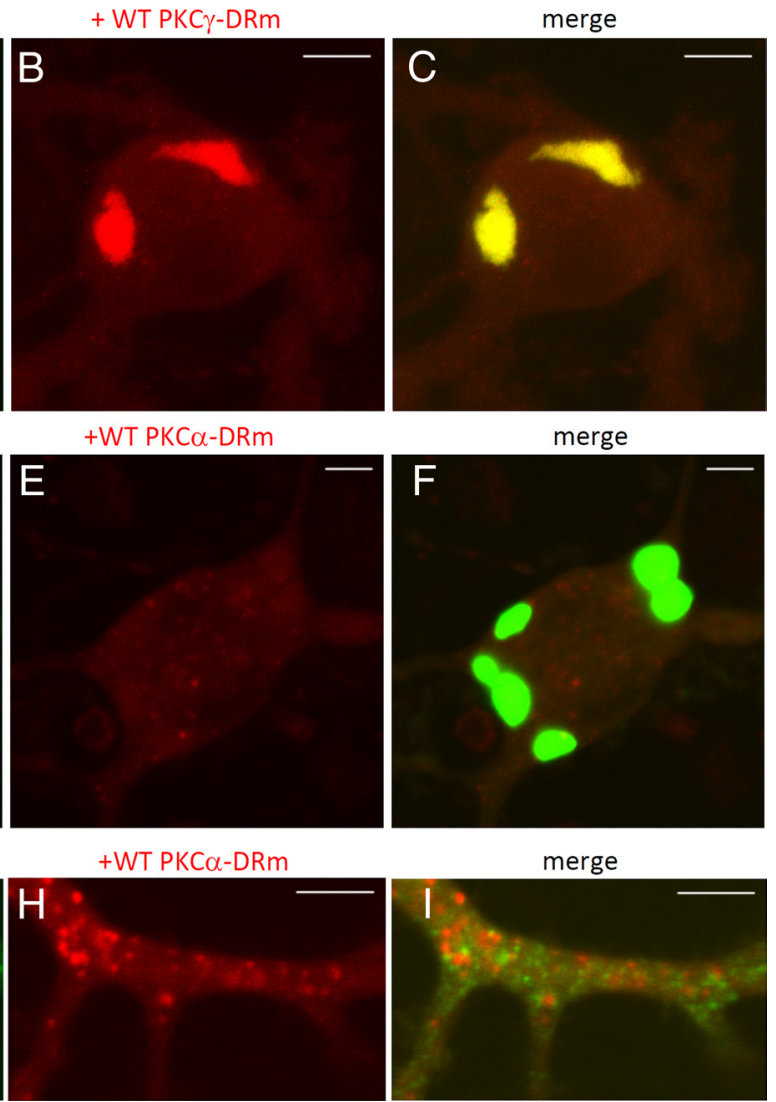

merge

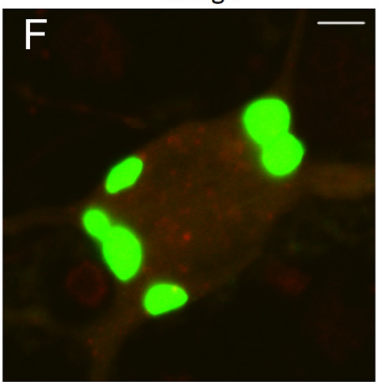

merge

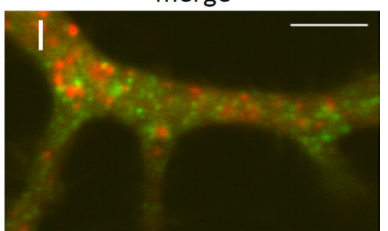

Figure 6. Colocalization and aggregation of wild-type PKC $\gamma$-DRm, but not wild-type PKC $\alpha$-DRm, with mutant PKC $\gamma$ GFP. A-C, Coexpression of mutant (Mu) PKC $\gamma$-GFP with wild-type (WT) PKC $\gamma$-DRm in cultured PCs. D-I, Coexpression of mutant (Mu) PKC $\gamma$-GFP with wild-type (WT) PKC $\alpha$-DRm in cultured PCs. Fluorescence images of GFP $(\boldsymbol{A}, \boldsymbol{D}, \boldsymbol{G})$, DsRed monomer $(\boldsymbol{B}, \boldsymbol{E}, \boldsymbol{H})$, and the superimposed images $(\boldsymbol{C}, \boldsymbol{F}, \boldsymbol{I})$ in the soma $(\boldsymbol{A}-\boldsymbol{F})$ and the dendritic shaft $(\boldsymbol{G}-\boldsymbol{I})$. Although PKC $\alpha$-DRm appears to form small aggregates in the dendrite $(\boldsymbol{H})$, they are in lysosomes; thus, they are not aggregates. Similar dots can be observed when only the DsRed monomer was expressed in cultured PCs. Scale bars: $\boldsymbol{A}-\boldsymbol{F}, 10 \mu \mathrm{m} ; \boldsymbol{G}-\boldsymbol{I}$, $5 \mu \mathrm{m}$.

mutant PKC $\gamma$-GFP could explain the failure of LTD expression. Thus, we coexpressed PKC $\alpha$-DRm and either wild-type or mutant PKC $\gamma$-GFP in cultured PCs and examined the translocation/recovery kinetics of PKC $\alpha$ and PKC $\gamma$ constructs upon depolarization with $100 \mathrm{~mm} \mathrm{KCl}$. Translocation of GFPor DRm-tagged constructs to the membrane was assessed by the percentage reduction of the cytoplasmic fluorescence intensity, and recovery kinetics were defined as the time required to achieve $50 \%$ recovery of the cytoplasmic fluorescence intensity after the maximal reduction of cytosolic fluorescence (half-time of fluorescence recovery; see Materials and Methods for measurement details).

$\mathrm{KCl}$ stimulation decreased the cytoplasmic GFP fluorescence intensity of wild-type PKC $\gamma$-GFP by almost $50 \%$, whereas the same stimulation decreased the fluorescence intensity of mutant PKC $\gamma$-GFP by only $\sim 17 \%$ (Fig. $7 A, C$ and supplemental Movies $1,2)$. In addition, the half-time of GFP fluorescence recovery was $19 \mathrm{~s}$ for wild-type PKC $\gamma$-GFP and only $6 \mathrm{~s}$ for mutant PKC $\gamma$-GFP (Fig. $7 A, D$ ). Notably, translocation of PKC $\alpha$-DRm to the membrane was not affected by the presence of mutant PKC $\gamma$-GFP (Fig. $7 B, E$ and supplemental Movies 3, 4); however, recovery of PKC $\alpha$-DRm from the membrane to the cytoplasm was significantly quicker in the presence of mutant PKC $\gamma$-GFP $(3 \mathrm{~s})$ than in the presence of wild-type PKC $\gamma$-GFP $(6 s)\left({ }^{* *} p<0.01\right.$ by unpaired $t$ test) (Fig. $7 B, F$ and supplemental Movies 3,4). These results suggest that translocated $\mathrm{PKC} \alpha$ cannot stay at the membrane long enough to phosphorylate substrates such as GluR2, and this may be the mechanism underlying the failure of LTD expression in mutant $\mathrm{PKC} \gamma$-GFP-expressing PCs in vivo.

\section{Discussion}

The present study showed that mutant PKC $\gamma$ formed numerous aggregates in PCs in vivo, similar to previous findings in primary cultured PCs (Seki et al., 2009). Patch-clamp analysis of PCs with aggregates revealed three abnormal phenotypes: (1) impaired elimination of surplus CF synapses from developing PCs; (2) disruption of LTD expression at PF-PC synapses; and (3) increased slow EPSC amplitude. In contrast to cultured PCs, neither maldevelopment of the dendrites nor apoptotic cell death was observed in PCs having aggregates in vivo, which was consistent with the electrophysiology results that failed to show significant alterations in the access resistance, passive membrane properties, basic excitatory synaptic transmission, or short-term synaptic plasticity at synapses between PFs and PCs with aggregates.

Only one postmortem brain of a SCA14 patient (H101Y mutation) has been examined by immunohistochemistry (Chen et al., 2003), and the SCA14 PCs showed markedly reduced immunoreactivity for PKC $\gamma$ without visible aggregation, in contrast to the present results showing marked aggregation of mutant PKC $\gamma$ in PCs. Because there was no information about the time between death and the fixation of the tissue, one possible explanation for the observed discrepancy in the aggregates is that they were degraded after death, which could explain the decreased immunoreactivity for PKC $\gamma$ in PCs in the postmortem sample.

To clarify the mechanisms underlying the impairment of redundant CF synapse elimination from developing PCs, we expressed wild-type PKC $\gamma$-DRm with mutant PKC $\gamma$-GFP in cultured PCs, because PKC $\gamma$ has been shown to be critical for CF synapse pruning from developing PCs (Kano et al., 1995). We found that mutant PKC $\gamma$-GFP formed aggregates with wild-type PKC $\gamma$-GFP in cultured PCs, which suggests that mutant PKC $\gamma$ functions in a dominant-negative manner. This idea was consistent with a previous study that used transgenic mice expressing a different mutant $(\mathrm{H} 101 \mathrm{Y})$ of $\mathrm{PKC} \gamma$ in which overexpression of mutant $\mathrm{PKC} \gamma(\mathrm{H} 101 \mathrm{Y})$ resulted in the malfunction of endogenous PKC $\gamma$ in PCs (Zhang et al., 2009). Thus, we believe that mutant PKC $\gamma$ from the affected allele disrupts the function of wild-type PKC $\gamma$ from the unaffected allele in PCs of SCA14 patients.

Failure of LTD expression in mutant PKC $\gamma$-GFP-expressing PCs was unexpected because PKC $\alpha$ is a key kinase for Ser880 phosphorylation of the AMPA receptor GluR2 during LTD in- 
duction (Leitges et al., 2004; Tanaka and Augustine, 2008). Although we did not find any aggregation of endogenous PKC $\alpha$ with mutant PKC $\gamma$, the membrane residence time of $\mathrm{PKC} \alpha$ after depolarization-induced membrane translocation was significantly decreased in the presence of mutant PKC $\gamma$, which could affect Ser880 phosphorylation of GluR2 at the postsynaptic membrane and disrupt LTD expression.

An important question that arose from the results was why the membrane residence time of PKC $\alpha$ decreased in the presence of mutant PKC $\gamma$. Membrane residence of $\mathrm{PKC}$ is regulated by diacylglycerol (DAG), and translocated PKC returns to the cytoplasm as the DAG level decreases. Diacylglycerol is converted to phosphatidic acid by diacylglycerol kinase $\gamma(\mathrm{DGK} \gamma)$ (Yamaguchi et al., 2006); thus, DGK $\gamma$ is a critical enzyme that regulates PKC dissociation from the membrane. Cytoplasmic DGK $\gamma$ is translocated to the membrane and activated through its phosphorylation by PKC $\gamma$ (Yamaguchi et al., 2006). Two independent studies have demonstrated that SCA14 mutations in PKC $\gamma$, including S119P, make PKC $\gamma$ constitutively active (Adachi et al., 2008; Verbeek et al., 2008). One intriguing hypothesis is that the constitutively active mutant PKC $\gamma$ would enhance the phosphorylation state of cytoplasmically localized DGK $\gamma$, which translocates to the membrane in response to extracellular stimuli, and thus would dysregulate the metabolism of DAG in the plasma membrane and decrease the membrane residence time of PKC $\alpha$ and PKC $\gamma$. This notion further supports the idea that SCA14 mutations pathologically affect PCs through a toxic gain-of-function and not a loss-of-function, which is consistent with previous reports showing robust LTD expression in PKC $\gamma$-knock-out PCs (Chen et al., 1995) and PCs with markedly diminished PKC $\gamma$ due to targeted RNA interference (Leitges et al., 2004). Indeed, the loss of PKC $\gamma$ affects DGK $\gamma$ activation and may result in prolonged localization of $\operatorname{PKC} \alpha$ at the membrane.

Stimulation of mGluR1 activates G protein-coupled phospholipase $\mathrm{C}$ to produce DAG and inositol triphosphate. The latter triggers $\mathrm{Ca}^{2+}$ release from the endoplasmic reticulum, which leads to the activation and membrane translocation of PKC. Diacylglycerol triggers gating of TRPC3 channels in PCs, which initiates cation influx and slow EPSCs. Notably, translocated wild-type PKC $\gamma$ phosphorylated TRPC3 channels and suppressed channel activity in COS-7 cells, whereas constitutively active mutant PKC $\gamma$, including the S119P mutant, failed to phosphorylate TRPC3 channels because of shorter membrane residence time (Adachi et al., 2008). Similarly, Verbeek et al. reported that mutant PKC $\gamma$ had reduced activity at the plasma membrane despite its constitutive activity in the cytoplasm (Verbeek et al., 2008). These findings suggest that both activity and membrane localization of PKC $\gamma$ are critical for phosphorylation of a membrane substrate such as TRPC3. In the present study, we showed interference of mutant PKC $\gamma$-GFP with wild-type PKC $\gamma$-GFP (Fig. 6A-C) and the impaired translocation and shorter membrane residence times of mutant PKC $\gamma$-GFP (Fig. $7 A, C, D)$, all of which result in a lower PKC $\gamma$ concentration at the membrane of PCs despite the constitutively active nature of the mutant construct. This fits with the finding of larger slow EPSC amplitudes in mutant PKC $\gamma$-GFP-expressing PCs. In contrast, wild-type PKC $\gamma$-GFP expression did not significantly decrease the amplitude of slow EPSCs. We hypothesize that phosphorylation of TRPC3 may be almost completely saturated by endogenous PKC activity. Further studies are needed to fully clarify the pathology of mutant PKC $\gamma$ in PCs.

As discussed above, the three abnormal phenotypes observed in PCs expressing mutant PKC $\gamma$-GFP can all be explained by dominant-negative actions of the mutant PKC $\gamma$ construct on endogenous $\gamma$ and $\alpha$ isoforms of PKC; thus, it is plausible that upstream mGluR1 activation is not impaired. This idea was supported by the finding that endocannabinoid-mediated presynaptic suppression was not affected by mutant PKC $\gamma$-GFP expression (supplemental Fig. 6). This short-term plasticity is an indirect assessment of mGluR1 activity, however, and we cannot completely exclude the possibility that the functional abnormalities caused by mutant PKC $\gamma$-GFP expression are partially due to an impairment of mGluR1 activation.

The present results show that mutant PKC $\gamma$-GFP expression disrupted LTD expression without degenerative signs in PCs, which prompts us to speculate that deficient cerebellar motor learning precedes PC degeneration in SCA14 patients. Very recently, using three LTD-deficient mutant mice, it has been demonstrated that PF-PC LTD is not essential for vestibulo-ocular reflex (VOR) adaptation, eyeblink conditioning, or locomotor learning (Schonewille et al., 2011). These three mutants exhibit abnormal AMPA receptor endocytosis at PF to PC synapses, which occur late in the LTD signaling cascade. However, PKC acts upstream of the cascade, 
and mutant PKC $\gamma$ expression could impair other forms of cerebellar plasticity such as presynaptic plasticity at the PC to cerebellar nuclei neuron synapse (Pedroarena and Schwarz, 2003) and/or postsynaptic plasticity at molecular layer interneurons to PC synapses (Song and Messing, 2005). Notably, VOR adaptation and delayed eyeblink conditioning are disrupted in mutant mice that express a PKC inhibitor peptide specifically in PCs (De Zeeuw et al., 1998; Goossens et al., 2001; Koekkoek et al., 2003). Thus, motor learning deficits may precede PC degeneration in SCA14 patients. It would be interesting to test whether delayed eyeblink conditioning, which is closely associated with cerebellar plasticity (Kishimoto et al., 2001a,b; Koekkoek et al., 2003), is impaired in carriers of the mutant SCA14 gene.

\section{References}

Adachi N, Kobayashi T, Takahashi H, Kawasaki T, Shirai Y, Ueyama T, Matsuda T, Seki T, Sakai N, Saito N (2008) Enzymological analysis of mutant protein kinase $\mathrm{C} \gamma$ causing spinocerebellar ataxia type 14 and dysfunction in $\mathrm{Ca}^{2+}$ homeostasis. J Biol Chem 283:19854-19863.

Brown SP, Brenowitz SD, Regehr WG (2003) Brief presynaptic bursts evoke synapse-specific retrograde inhibition mediated by endogenous cannabinoids. Nat Neurosci 6:1048-1057.

Carlson KM, Andresen JM, Orr HT (2009) Emerging pathogenic pathways in the spinocerebellar ataxias. Curr Opin Genet Dev 19:247-253.

Chen C, Kano M, Abeliovich A, Chen L, Bao S, Kim JJ, Hashimoto K, Thompson RF, Tonegawa S (1995) Impaired motor coordination correlates with persistent multiple climbing fiber innervation in PKC gamma mutant mice. Cell 83:1233-1242.

Chen DH, Brkanac Z, Verlinde CL, Tan XJ, Bylenok L, Nochlin D, Matsushita M, Lipe H, Wolff J, Fernandez M, Cimino PJ, Bird TD, Raskind WH (2003) Missense mutations in the regulatory domain of PKC $\gamma$ : a new mechanism for dominant nonepisodic cerebellar ataxia. Am J Hum Genet 72:839-849.

Chung HJ, Steinberg JP, Huganir RL, Linden DJ (2003) Requirement of AMPA receptor GluR2 phosphorylation for cerebellar long-term depression. Science 300:1751-1755.

De Zeeuw CI, Hansel C, Bian F, Koekkoek SK, van Alphen AM, Linden DJ, Oberdick J (1998) Expression of a protein kinase C inhibitor in Purkinje cells blocks cerebellar LTD and adaptation of the vestibulo-ocular reflex. Neuron 20:495-508.

Goossens J, Daniel H, Rancillac A, van der Steen J, Oberdick J, Crépel F, De Zeeuw CI, Frens MA (2001) Expression of protein kinase C inhibitor blocks cerebellar long-term depression without affecting Purkinje cell excitability in alert mice. J Neurosci 21:5813-5823.

Hartmann J, Dragicevic E, Adelsberger H, Henning HA, Sumser M, Abramowitz J, Blum R, Dietrich A, Freichel M, Flockerzi V, Birnbaumer L, Konnerth A (2008) TRPC3 channels are required for synaptic transmission and motor coordination. Neuron 59:392-398.

Hirai H (2001) Modification of AMPA receptor clustering regulates cerebellar synaptic plasticity. Neurosci Res 39:261-267.

Hirai H, Pang Z, Bao D, Miyazaki T, Li L, Miura E, Parris J, Rong Y, Watanabe M, Yuzaki M, Morgan JI (2005) Cbln1 is essential for synaptic integrity and plasticity in the cerebellum. Nat Neurosci 8:1534-1541.

Iizuka A, Takayama K, Torashima T, Yamasaki M, Koyama C, Mitsumura K, Watanabe M, Hirai H (2009) Lentiviral vector-mediated rescue of motor behavior in spontaneously occurring hereditary ataxic mice. Neurobiol Dis 35:457-465.

Kano M, Hashimoto K (2009) Synapse elimination in the central nervous system. Curr Opin Neurobiol 19:154-161.

Kano M, Hashimoto K, Chen C, Abeliovich A, Aiba A, Kurihara H, Watanabe M, Inoue $\mathrm{Y}$, Tonegawa S (1995) Impaired synapse elimination during cerebellar development in PKC gamma mutant mice. Cell 83:1223-1231.

Kishimoto Y, Kawahara S, Suzuki M, Mori H, Mishina M, Kirino Y (2001a) Classical eyeblink conditioning in glutamate receptor subunit delta $2 \mathrm{mu}-$ tant mice is impaired in the delay paradigm but not in the trace paradigm. Eur J Neurosci 13:1249-1253.

Kishimoto Y, Kawahara S, Fujimichi R, Mori H, Mishina M, Kirino Y (2001b) Impairment of eyeblink conditioning in GluRdelta2-mutant mice depends on the temporal overlap between conditioned and unconditioned stimuli. Eur J Neurosci 14:1515-1521.

Koekkoek SK, Hulscher HC, Dortland BR, Hensbroek RA, Elgersma Y, Ruigrok TJ, De Zeeuw CI (2003) Cerebellar LTD and learningdependent timing of conditioned eyelid responses. Science 301:17361739.

Leitges M, Kovac J, Plomann M, Linden DJ (2004) A unique PDZ ligand in PKCalpha confers induction of cerebellar long-term synaptic depression. Neuron 44:585-594.

Maejima T, Hashimoto K, Yoshida T, Aiba A, Kano M (2001) Presynaptic inhibition caused by retrograde signal from metabotropic glutamate to cannabinoid receptors. Neuron 31:463-475.

Marcaggi P, Attwell D (2005) Endocannabinoid signaling depends on the spatial pattern of synapse activation. Nat Neurosci 8:776-781.

Marcaggi P, Attwell D (2007) Short- and long-term depression of rat cerebellar parallel fibre synaptic transmission mediated by synaptic crosstalk. J Physiol 578:545-550.

Matsuda S, Mikawa S, Hirai H (1999) Phosphorylation of serine-880 in GluR2 by protein kinase $\mathrm{C}$ prevents its $\mathrm{C}$ terminus from binding with glutamate receptor-interacting protein. J Neurochem 73:1765-1768.

Matsuda S, Launey T, Mikawa S, Hirai H (2000) Disruption of AMPA receptor GluR2 clusters following long-term depression induction in cerebellar Purkinje neurons. EMBO J 19:2765-2774.

Nishizuka Y (1992) Intracellular signaling by hydrolysis of phospholipids and activation of protein kinase C. Science 258:607-614.

Niwa H, Yamamura K, Miyazaki J (1991) Efficient selection for highexpression transfectants with a novel eukaryotic vector. Gene 108:193199.

Pedroarena CM, Schwarz C (2003) Efficacy and short-term plasticity at GABAergic synapses between Purkinje and cerebellar nuclei neurons. J Neurophysiol 89:704-715.

Safo PK, Regehr WG (2005) Endocannabinoids control the induction of cerebellar LTD. Neuron 48:647-659.

Saito N, Kikkawa U, Nishizuka Y, Tanaka C (1988) Distribution of protein kinase C-like immunoreactive neurons in rat brain. J Neurosci 8:369-382.

Sakai N, Sasaki K, Ikegaki N, Shirai Y, Ono Y, Saito N (1997) Direct visualization of the translocation of the gamma-subspecies of protein kinase $\mathrm{C}$ in living cells using fusion proteins with green fluorescent protein. J Cell Biol 139:1465-1476.

Schonewille M, Gao Z, Boele HJ, Veloz MF, Amerika WE, Simek AA, De Jeu MT, Steinberg JP, Takamiya K, Hoebeek FE, Linden DJ, Huganir RL, De Zeeuw CI (2011) Reevaluating the role of LTD in cerebellar motor learning. Neuron 70:43-50.

Schrenk K, Kapfhammer JP, Metzger F (2002) Altered dendritic development of cerebellar Purkinje cells in slice cultures from protein kinase C $\gamma$-deficient mice. Neuroscience 110:675-689.

Seki T, Adachi N, Ono Y, Mochizuki H, Hiramoto K, Amano T, Matsubayashi H, Matsumoto M, Kawakami H, Saito N, Sakai N (2005) Mutant protein kinase Cgamma found in spinocerebellar ataxia type 14 is susceptible to aggregation and causes cell death. J Biol Chem 280:29096-29106.

Seki T, Takahashi H, Adachi N, Abe N, Shimahara T, Saito N, Sakai N (2007) Aggregate formation of mutant protein kinase C gamma found in spinocerebellar ataxia type 14 impairs ubiquitin-proteasome system and induces endoplasmic reticulum stress. Eur J Neurosci 26:3126-3140.

Seki T, Shimahara T, Yamamoto K, Abe N, Amano T, Adachi N, Takahashi H, Kashiwagi K, Saito N, Sakai N (2009) Mutant gammaPKC found in spinocerebellar ataxia type 14 induces aggregate-independent maldevelopment of dendrites in primary cultured Purkinje cells. Neurobiol Dis 33:260-273.

Seki T, Abe-Seki N, Kikawada T, Takahashi H, Yamamoto K, Adachi N, Tanaka S, Hide I, Saito N, Sakai N (2010) Effect of trehalose on the properties of mutant \{gamma\}PKC, which causes spinocerebellar ataxia type 14, in neuronal cell lines and cultured Purkinje cells. J Biol Chem 285:33252-33264.

Song M, Messing RO (2005) Protein kinase $C$ regulation of $\mathrm{GABA}_{\mathrm{A}}$ receptors. Cell Mol Life Sci 62:119-127.

Tanaka K, Augustine GJ (2008) A positive feedback signal transduction loop determines timing of cerebellar long-term depression. Neuron 59:608-620. 
Torashima T, Okoyama S, Nishizaki T, Hirai H (2006a) In vivo transduction of murine cerebellar Purkinje cells by HIV-derived lentiviral vectors. Brain Res 1082:11-22.

Torashima T, Yamada N, Itoh M, Yamamoto A, Hirai H (2006b) Exposure of lentiviral vectors to subneutral $\mathrm{pH}$ shifts the tropism from Purkinje cell to Bergmann glia. Eur J Neurosci 24:371-380.

Torashima T, Koyama C, Iizuka A, Mitsumura K, Takayama K, Yanagi S, Oue M, Yamaguchi H, Hirai H (2008) Lentivector-mediated rescue from cerebellar ataxia in a mouse model of spinocerebellar ataxia. EMBO Rep 9:393-399.

Verbeek DS, Goedhart J, Bruinsma L, Sinke RJ, Reits EA (2008) PKC gamma mutations in spinocerebellar ataxia type 14 affect $\mathrm{C} 1$ domain accessibility and kinase activity leading to aberrant MAPK signaling. J Cell Sci 121:2339-2349.
Xia J, Chung HJ, Wihler C, Huganir RL, Linden DJ (2000) Cerebellar longterm depression requires PKC-regulated interactions between GluR2/3 and PDZ domain-containing proteins. Neuron 28:499-510.

Yamaguchi Y, Shirai Y, Matsubara T, Sanse K, Kuriyama M, Oshiro N, Yoshino K, Yonezawa K, Ono Y, Saito N (2006) Phosphorylation and upregulation of diacylglycerol kinase gamma via its interaction with protein kinase C gamma. J Biol Chem 281:31627-31637.

Yamamoto K, Seki T, Adachi N, Takahashi T, Tanaka S, Hide I, Saito N, Saka N (2010) Mutant protein kinase C gamma that causes spinocerebellar ataxia type 14 (SCA14) is selectively degraded by autophagy. Genes Cells $15: 425-438$.

Zhang Y, Snider A, Willard L, Takemoto DJ, Lin D (2009) Loss of Purkinje cells in the PKC $\gamma$ H101Y transgenic mouse. Biochem Biophys Res Commun 378:524-528. 